\title{
Costituzione e mutamento. Le dinamiche della cittadinanza in età moderna e contemporanea
}

di Marco Fioravanti

Dic 9, 2020 | Emergenza e (nuova) normalità, In evidenza $|\underline{0}|$

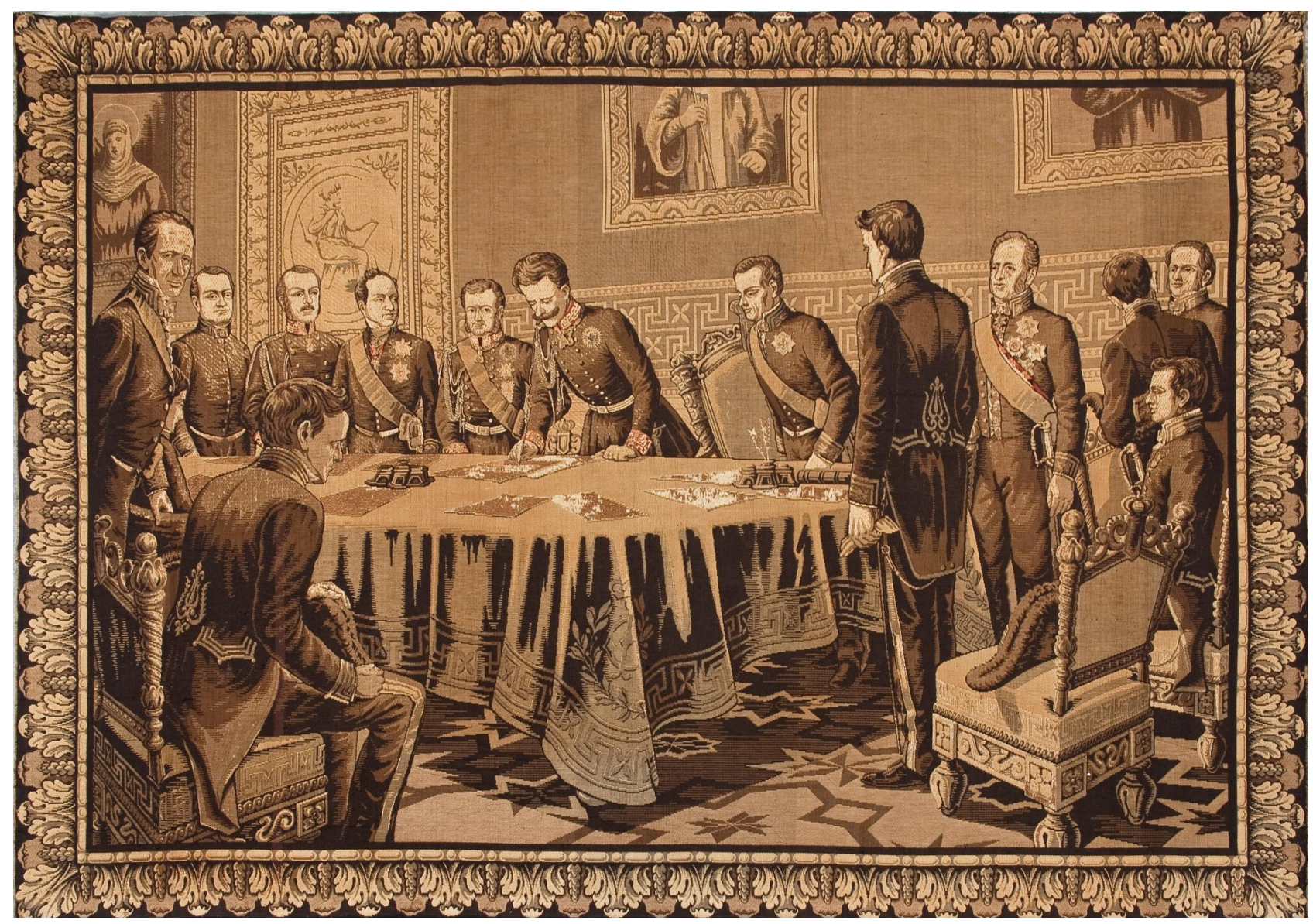

Carlo Alberto firma lo Statuto il 4 marzo 1848

\section{Abstract}

La cittadinanza moderna, riconducibile alle rivoluzioni borghesi sei-settecentesche, nasce dall'idea di un'eguaglianza naturale nei diritti. Sia la Rivoluzione americana che quella francese sono accomunate dalla ricerca di un ordinamento giuridico che potesse garantire libertà, proprietà e sicurezza. A partire dalla seconda metà dell'Ottocento, però, il modello liberale di cittadinanza/rappresentanza fatica a gestire l'ascesa delle masse sulla scena politica.

La Prima guerra mondiale ha come effetto l'allargamento dei confini della cittadinanza, avvenuto principalmente attraverso la conquista del suffragio universale maschile e l'avvento dei partiti politici di massa. Conclusasi, dopo la Seconda guerra mondiale, la fase dei fascismi in Europa, la rappresentanza trova una nuova dimensione grazie all'azione dei partiti politici di massa.

L'articolo si propone di sintetizzare tale processo di lungo periodo in funzione di un 
approfondimento sulla Costituzione Italiana che risulta di grande utilità per i nuovi percorsi dell'insegnamento di Educazione Civica.

\author{
«La patria non può sussistere senza la libertà, \\ né la libertà senza la virtù, né la virtù senza cittadini: \\ avrete tutto se formate dei cittadini; \\ senza di questo non avrete che cattivi schiavi, \\ a cominciare dai capi di stato», \\ Jean-Jacques Rousseau, Économie politique.
}

\title{
Rivoluzione e Costituzione
}

La nascita della cittadinanza moderna, come è noto riconducibile alle rivoluzioni borghesi seisettecentesche, muove dal presupposto di un'eguaglianza naturale nei diritti. Sia la Rivoluzione americana che quella francese, diverse per tanti aspetti dal punto di vista politico-costituzionale, sono accomunate dalla ricerca di un ordinamento giuridico che potesse garantire libertà, proprietà e sicurezza. Nel caso delle colonie americane la necessità di svincolarsi dalla madrepatria spinse $\mathrm{i}$ framers a cercare una soluzione istituzionale che consolidasse il sistema di common law "innestato" nel Nuovo Mondo. Non a caso si è parlato di rivoluzione conservatrice per indicare uno stravolgimento che mirava a ripristinare i principi giuridici della tradizione inglese, violati proprio da Giorgio III. In particolare il "grimaldello" tecnico utilizzato dai costituenti americani fu proprio il principio secondo cui coloro che pagano le tasse devono contribuire alla scelta dei rappresentanti, idealmente riconducibile alla magna carta libertatum, e riassumibile con la nota espressione anglosassone: no taxation without representation. In tal modo si affermava il connubio, risalente a sua volta alle rivoluzioni inglesi del Seicento, che legava la cittadinanza alla proprietà.

Percorso simile, in un contesto fortemente diverso, fu intrapreso dai rivoluzionari francesi i quali, soprattutto in una prima fase, si videro spinti dalla necessità di superare le distinzioni di status che avevano caratterizzato la società d'Ancien régime e dal bisogno di portare a compimento un progetto che risaliva, per paradossale che possa apparire, proprio all'assolutismo: l'uguaglianza dei cittadini (non più sudditi) di fronte alla legge e la liberazione della proprietà dai vincoli feudali. Il "Prometeo liberato" dalla rivoluzione industriale continuava la sua ascesa senza limiti verso vette ancora più alte...fino tuttavia a costruire nuove e forse più stringenti catene.

Il mutato clima istituzionale e il protagonismo delle classi subalterne, portò repentinamente a un cambio di paradigma, quando, grazie anche all'esperienza americana e alla concettualizzazione dell'abate Sieyès, fu azionato il potere costituente, un dispositivo che capovolgeva la gerarchia dei poteri e contribuiva a ridisegnare lo spazio pubblico. La Dichiarazione dei diritti dell'uomo e del cittadino del 26 agosto 1789 rappresenta sia la fine di un percorso di matrice giusnaturalistica e di superamento dei privilegi, sia l'inizio di un'epoca nuova, l'età dei diritti, che avrebbe trovato il suo pendant civilistico con l'emanazione nel 1804 del Code civil des Français.

L'epoca nuova che si andava dischiudendo, edificata su libertà e proprietà, si basava sulla distinzione, per quanto artificiosa, tra cittadini attivi e passivi: i primi titolari di diritti politici, in primis il diritto di voto, i secondi di diritti civili, come le garanzie giurisdizionali. Fu solo con la fase più radicale della Rivoluzione francese che si ottennero delle conquiste che svincolarono per la prima volta l'appartenenza alla pólis dalla proprietà, legandola semplicemente al lavoro e alla nascita sul territorio francese. La costituzione giacobina del 1793 comportò tali acquisizioni - come 
il suffragio universale maschile, il diritto al lavoro, alla salute e all'educazione, la brevità del mandato, il diritto di resistenza - che si sarebbe dovuto attendere la costituzione di Weimar del 1919 per incontrare un costituzionalismo così avanzato. Ma sia il riformismo del biennio 1789-1791 che il radicalismo dell'anno primo vennero meno durante il periodo direttoriale e con l'ascesa di Napoleone, che, a sua volta, seppe mitigare le riforme rivoluzionarie recuperando istituzioni e prassi di Antico regime. Con la Restaurazione poi si affermò un costituzionalismo duale, espressione del compromesso tra borghesia e aristocrazia, sfociato nelle Charte del 1814 e del 1830, le quali rappresentavano, insieme al Code Napoléon, il trionfo della borghesia e inauguravano quell'idea capacitaria di cittadinanza il cui alfiere fu François Guizot.

\section{Cittadinanza e democrazia}

Il 1848 europeo mise in discussione il paradigma proprietario e l'ordine censitario a favore di una partecipazione popolare che trovò nelle forme embrionali di organizzazione partitica, nel mutualismo, nell'associazionismo i nuovi soggetti storici del cambiamento. Sebbene in tutta Europa le rivoluzioni di metà Ottocento incontrarono presto il loro Termidoro, il segno lasciato dai rivolgimenti popolari e proto-proletari aprì un processo che condizionò le scelte politiche delle classi dirigenti europee. La Comune di Parigi del 1871, canto del cigno di un movimento di popolo che voleva autogovernarsi riattivando meccanismi di controllo del potere e di partecipazione diretta alla vita politica, cercò nella dimensione municipale la misura per pensare la democrazia. Quanto questa esperienza, rimossa o demonizzata, abbia influito sulle future scelte politiche e come abbia rappresentato il simbolo dell'emancipazione meriterebbe ancora nuovi studi.

Il modello liberale del cosiddetto "governo dei migliori" [1], basato sull'idea che l'élite del Paese essenzialmente i proprietari - scegliessero i rappresentanti più validi che avevano il compito di controllare il potere, attraverso la finzione della rappresentanza ${ }^{[2]}$, andò incontro alla propria sconfitta quando non seppe gestire l'ascesa delle masse sulla scena politica. La rappresentanza si ridusse pertanto, per lo meno a partire dalla fine della Seconda repubblica francese, a mera finzione, che nascondeva l'acerrima volontà di arginare l'ingresso del popolo nei confini della cittadinanza. La delega rappresentativa si trasformò, secondo l'efficace espressione di Pierre Bourdieu, in una forma di feticismo politico ${ }^{[3]}$.

La società borghese, che vide nella Belle époque il suo mesto destino di apparente gaiezza e abbondanza, trovò nelle trincee della prima guerra mondiale la sua nemesi e, come conseguenza, proprio l'allargamento dei confini della cittadinanza, avvenuto principalmente attraverso la conquista del suffragio universale maschile, con la conseguente legittimazione dei partiti politici di massa. La rivoluzione sovietica e la Repubblica di Weimar rappresentano a loro volta due momenti, simili per presupposti ma distanti nelle prospettive, di superamento del costituzionalismo borghese a favore di una democratizzazione del sistema. Con esiti, tuttavia, sebbene in contesti e per motivi diversi, disastrosi.

\section{Crisi dello ius publicum europaeum}

La crisi di quello che tradizionalmente definiamo ius publicum europeaum, a cavaliere tra il XIX e il XX secolo, fu caratterizzata da mutamenti sociali e istituzionali tali da mettere in discussione un assetto consolidatosi dalle rivoluzioni borghesi settecentesche. Con il lento passaggio dallo Stato monoclasse a quello pluriclasse - secondo la celebre distinzione di Massimo Severo Giannini - il 
percorso del costituzionalismo liberale, basato su una rappresentanza "virtuale", fu interrotto dalla crisi innescata, nel sistema costituzionale italiano ed europeo, dall'ampliamento delle basi della partecipazione politica nella società di massa, dall'affermarsi di un'embrionale struttura partitica e dalla radicalizzazione dello scontro di classe.

Nell'entre deux guerres era stato acceso il dibattito tra i giuristi europei sulla necessità di superare il tradizionale modello liberale, espressione in estrema sintesi della tradizione rivoluzionaria francese, per lo meno nella sua variante moderata, a vantaggio di una diversa concezione della rappresentanza e più in generale del rapporto tra governanti e governati, oltre che, in ultima istanza, del ruolo dello Stato.

Il fascismo interruppe questo processo, che seppure tra molti limiti e contraddizioni aveva avviato un ripensamento dei limiti del modello liberale e la necessità di democratizzare la società aprendo verso istanze pluraliste. Il regime invece, frutto avvelenato della prima guerra mondiale, riuscì ad assorbire le critiche e gli umori antiparlamentari in un'ottica autoritaria e totalitaria, che trovò nella concettualizzazione del giurista weimariano Ernst Fraenkel del doppio Stato una sua chiara interpretazione: così come era avvenuto per il regime nazista, nell'Italia fascista, seppure in misura diversa, a fianco di una legalità formale sempre più marginale si affermava prepotentemente uno stato d'eccezione che lasciava ampio spazio di manovra all'amministrazione ${ }^{[4]}$.

\section{Diritto e Resistenza in Italia}

Nella stagione resistenziale e negli anni della Costituente, i nuovi protagonisti non si limitarono a riprendere il discorso su diritto e Stato interrotto violentemente con il fascismo, ma ripensarono le categorie del diritto in un assetto dei poteri in trasformazione e in fermento. Detto in altri termini si cercò di allargare le maglie strette in cui lo Stato di diritto aveva operato e di restringere quel divario tra Stato e società che aveva caratterizzato la dottrina liberale.

L'anteriorità dei diritti della persona rispetto allo Stato fu al centro del dibattito post-resistenziale, soprattutto nel mondo cattolico. Critica verso il positivismo e lo statalismo, infatti, fu la corrente di giuristi cattolici rappresentati, nella fase resistenziale, emblematicamente da Giorgio la Pira e Giuseppe Dossetti. Il primo mosso da un fortissimo afflato metafisico e teologico, il secondo più attento alle questioni terrene, ma spinto da una prospettiva tomista. Sebbene tra loro molto diversi, i due giuristi cattolici - romanista il primo, canonista il secondo - erano accomunati da una avversione, di stampo chiaramente cristiano, non solo verso il lascito ideologico del fascismo, ma anche nei confronti dei limiti dello Stato liberale. Secondo Dossetti quest'ultimo, che aveva disconosciuto i corpi intermedi e i diritti delle persone nella loro individualità, aveva la grande debolezza di essere privo di un programma e di un fine. Sia per La Pira che per Dossetti le fondamenta dello Stato democratico avrebbero dovuto basarsi sul riconoscimento e la garanzia dei diritti sacri, naturali e inalienabili del cittadino. L'edificio costituzionale da essi ipotizzato, di stampo personalista e pluralista, riconosceva l'anteriorità della persona (e dei suoi diritti) sullo Stato. La lotta per il riconoscimento delle comunità intermedie, in controtendenza rispetto alla tradizione giuridica liberale di derivazione francese, trovò una sua alta espressione anche nell'opera di Costantino Mortati, che vedeva in esse un elemento di forza per lo Stato e non di debolezza.

Nella prospettiva di rottura con la tradizione giuridica positivistica e in continuità con una visione etico-religiosa di chiara impronta tomista, si può leggere una delle pagine più avvincenti dell'esperienza costituente, ovvero la proposta di Dossetti dell'inserimento in costituzione del diritto di resistenza, che rappresenta la misura "ultima" dei confini della sovranità. Premesso che si 
tratta di un diritto azionabile in casi di estrema crisi dell'ordinamento quando le vie legali tradizionali sono di fatto o di diritto precluse, esso tuttavia rimane un diritto tanto affascinante quanto controverso il cui richiamo ha sempre destato perplessità tra i giuristi. L'articolato presentato da Dossetti, che riprendeva l'art. 35 della Dichiarazione dei diritti giacobina del 1793, stabiliva, al II comma dell'art. 50 del Progetto di Costituzione, che "La resistenza individuale e collettiva agli atti dei pubblici poteri, che violino le libertà fondamentali e i diritti garantiti dalla presente Costituzione, è diritto e dovere di ogni cittadino". La Costituente, com'è noto, respinse l'inserimento in Costituzione di questo diritto e in particolare Mortati vi si oppose in quanto, sebbene si ricollegasse alla tradizione cattolica, rivestiva un carattere metagiuridico.

Il diritto di resistenza avrebbe trovato nelle prassi comunicative, nel principio di opposizione, nelle risorse di contropotere e più in generale nel potere negativo una sua forma all'altezza dello Stato costituzionale. Lasciando intravedere in controluce che il problema del potere si esaurisce nei contropoteri, quasi per sottrazione, e che la Resistenza, così come il potere costituente (che di essa ne è la veste giuridica) è un'azione che si sottrae al dominio del diritto ${ }^{[5]}$. Il diritto di resistenza inteso dunque come "risorsa inventiva per la politica", secondo la bella definizione di Donatella Di Cesare[6].

Ma l'eredità che l'esperienza resistenziale ha lasciato per la costituzione repubblicana non si può ridurre ai lavori dell'Assemblea costituente o ai progetti di costituzione di singoli individui, anche se rilevanti. Essa consiste invero in tutta quella attività delle Repubbliche partigiane $\mathrm{e}$ nell'esperienza dei CLN Alta Italia che hanno contribuito ad anticipare i lavori della Costituente, attraverso regole scritte, consuetudini e convenzioni costituzionali. Come dimostrato recentemente da Giuseppe Filippetta ${ }^{[7]}$ e come ricordato, con sensibilità diverse, da Mario Dogliani $\left[\frac{[8]}{\text {, la }}\right.$ Costituzione nacque anche nelle bande partigiane. Si tratta di "anticipazioni della Costituzione" dove in un contesto difficile e drammatico come quello della guerra civile si elaborarono idee di democrazia basata sull'eguaglianza dei diritti politici, sull'universalità del suffragio maschile e femminile, sulla necessità del consenso popolare per ogni scelta politica, sia del governo locale che di quello nazionale. Si proposero interventi nell'economia per tutelare i redditi più bassi, politiche fiscali orientate alla progressività delle imposte, assistenza sanitaria gratuita, riforme dell'amministrazione della giustizia in chiave garantista, scuola media unificata, Università popolari, stampa libera. La Resistenza dunque azionò meccanismi di produzione sociale e giuridica svincolati anche dall'autosufficienza dei partiti.

\section{La Repubblica dei partiti e la crisi della rappresentanza}

Con l'ascesa delle masse sull'agone politico, con il superamento del sistema liberal-censitario e con la fine dei fascismi in Europa, dopo la Seconda guerra mondiale la rappresentanza sarebbe stata filtrata dai partiti politici di massa che mediando con la società si sarebbero fatti latori di una rappresentanza degli interessi, cambiando pertanto la natura della rappresentanza stessa ${ }^{[9]}$. Se per un verso la Repubblica dei partiti ha cercato di completare e integrare la rappresentanza, attenuando lo iato tra istituzioni e popolo (obiettivo tuttavia non del tutto raggiunto), per un altro la loro funzione principale è sembrata quella semmai della sospensione della rappresentanza a fini democratici attraverso la disciplina (e il mandato) di partito ${ }^{[10]}$.

La cittadinanza repubblicana espressione delle lotte del movimento operaio, delle rivendicazioni del mondo cattolico e delle istanze più avanzate della liberal democrazia, trovò negli istituti della partecipazione popolare la sua più esplicita realizzazione. Tuttavia le conquiste dei cosiddetti Trenta gloriosi, dalla fine della seconda guerra mondiale alla metà degli anni Settanta, non devono 
offuscare un quadro che a una lettura più attenta risulta meno nitido. Le nuove coordinate della cittadinanza si iscrivono in un contesto, seppure tendenzialmente progressivo, di limitazioni e esclusioni soprattutto per le classi subalterne.

Inoltre le istituzioni del fordismo - lo stato sociale, il lavoro, la socialdemocrazia - sono state messe fortemente in discussione negli ultimi quarant'anni, caratterizzati da quella rivoluzione neoliberale che ha fatto saltare il compromesso tra capitale e lavoro alla base delle costituzioni europee del dopoguerra, comportando la perdita progressiva della centralità del progetto democratico che animava la Costituente.

La partecipazione democratica stessa ha vissuto un'involuzione progressiva giunta alla quasi totale disaffezione verso la politica dei cittadini e verso una forma estrema e sembrerebbe inedita di crisi della rappresentanza che ha mostrato tutta la vulnerabilità delle istituzioni rappresentative. Queste ultime, private del supporto dal basso mediato dai partiti politici, sono ridotte a gusci vuoti. E il vuoto tuttavia viene riempito in maniere diverse, molteplici e discordanti: da un lato dal populismo sovranista (e razzista) che, aggiornando la retorica nazionalista ottocentesca ${ }^{[11]}$, si autoproclama alfiere della disaffezione democratica, esasperando i risentimenti e la lotta degli/tra gli esclusi; da un altro lato, meno sconsolante, le piazze vengono riempite, a volte occupate, come luoghi "altri" della rappresentanza, come istanze alternative al potere e come esercizio della cittadinanza. Si pensi a Zuccotti Park, a Piazza Taksim, alle Nuit Debout, a Puerta del Sol, e a molti altri spazi globali che uniscono individualità diversissime tra loro, ma accomunate dal non sentirsi rappresentate e in cerca di nuove forme di partecipazione.

Tornando in Italia e agli aspetti maggiormente istituzionali, la sfaldatura degli equilibri costituzionali ha comportato in particolare l'affievolimento del principio di eguaglianza, contenuto nell'art. $3^{[12]}$. Esso, come ha scritto con la consueta eleganza Mario Dogliani, contiene in sé una «eccedenza di significato» ${ }^{[13]}$, ovvero incarna la natura rivoluzionaria della Costituzione, alla base di una prospettiva di riscatto, che il trentennio alle spalle sembra aver annichilito. Un'eccedenza che vale, ai nostri occhi, per tutta la Costituzione, non solo per i principi fondamentali. Nel suo insieme essa rappresenta un tutto unitario, nel senso che ogni articolo andrebbe letto alla luce dei primi dodici che forniscono "un'evidenza grafica" al nuovo ordinamento ${ }^{[14]}$. Anzi si è assistito a un processo di riforma della Costituzione che di fatto andava surrettiziamente a modificare i principi presenti nella parte dedicata ai diritti fondamentali, non modificabili nel loro "contenuto essenziale". Secondo la nota sentenza della Corte costituzionale n. 1146 del 1988 infatti «la Costituzione italiana contiene alcuni principi supremi che non possono essere sovvertiti o modificati nel loro contenuto essenziale neppure da leggi di revisione costituzionale ... Tali sono tanto i principi che la Costituzione esplicitamente prevede come limiti assoluti al potere di revisione costituzionale, quale la forma repubblicana (art. 139 Cost.), quanto i principi che, pur non essendo espressamente menzionati fra quelli non assoggettabili al procedimento di revisione costituzionale, appartengono all'essenza stessa dei valori supremi sui quali si fonda la Costituzione italiana»

Il principale difetto del riformismo costituzionale è stato proprio l'idea di una possibile riforma di una parte, di uno o più articoli, senza la capacità di una prospettiva teorica di ampio respiro e di lunga durata ${ }^{[16]}$. Di fronte alla "statica" delle tentate riforme costituzionali si profila la "dinamica" del mutamento costituzionale. In altri termini la Costituzione (quella italiana, ma più in generale "la costituzione dei moderni") è caratterizzata proprio da una logica "dinamica", ovvero basata sui rapporti politici e sulla dimensione storica, sul "punto e la linea", seguendo le intuizioni rispettivamente di Stefano Rodotà e Paolo Grossi. 
Il titolo di questo paragrafo conclusivo allude al bellissimo documentario The spirit of ' 45 (Ken Loach, 2013) nel quale è chiara la funzione storica di un mutamento che si radica nello spirito del tempo per concretizzarsi nel reale. E lo stesso, o qualcosa di simile, si potrebbe pensare riguardo il nostro 1948.

Il costituzionalismo moderno - nato come assiologico e prescrittivo in opposizione a quello degli antichi, di per sé avalutativo e descrittivo ${ }^{[17]}$ - richiede il mutamento, esige la trasformazione, ma nello spirito della costituzione stessa, alla luce del principio democratico, evitando ciò che Pietro Scoppola ha definito l'illusione di un perenne nuovo inizio ${ }^{[18]}$. La Costituzione - quell' «obbligo preventivo» di cui ha parlato Jon Elster ${ }^{[19]}$ - è un progetto che trova in quell'eccedenza democratica ed egualitaria la ragione della sua continua attuazione.

Il principio di eguaglianza, definito come "un dover essere", ha trovato nuove forme (non sempre progressive) di interpretazione a livello della giurisprudenza europea ${ }^{[20]}$. Dopo una lunga fase di riforme (o controriforme) neoliberali si è di fatto affermata una nuova costituzione economica - o se vogliamo una costituzione materiale del neoliberismo ${ }^{[21]}$ - che in Italia si profila in antitesi rispetto a quella tracciata dall'Assemblea costituente ${ }^{[22]}$ e che trova nella riforma dell'articolo 81 un equivoco punto di arrivo. Per contro questa nuova "grande trasformazione" ha rilanciato negli ultimi anni una stagione di ricerche giuridiche, ricostruzioni storiche e interpretazioni filosofiche, tutte attente alla dimensione costituzionale del "diritto dei privati"[23], alla dinamica dei beni comuni e alla "caratura costituzionale" delle proprietà collettive.

Il quarantennio di dominio neoliberista, attraverso l'appropriazione degli strumenti intellettuali dei suoi storici avversari, ha colpito non solo le istituzioni del welfare alla base del compromesso fordista o socialdemocratico, ma ha preso di mira, pour cause, soprattutto il campo dell'educazione attraverso spinte concentriche alla privatizzazione, alla valorizzazione di un sedicente capitale umano in ogni aspetto dell'istruzione, attraverso le famigerate agenzie di rating, utilizzate in qualunque ambito della vita: la biopolitica neoliberale arriva in ambiti e spazi che il liberalismo tradizionale aveva lasciato intatti $[24]$.

Tutto ciò ci spinge a un ripensamento della fallimentare stagione delle riforme costituzionali alla luce della consapevolezza che non è nelle riforme grandi o piccole l'unica strada da seguire, ma nel considerare la Costituzione come punto di partenza, non certo di arrivo, come una rivoluzione in cammino, o come una rivoluzione promessa, secondo la celebre definizione, a volte un po' abusata retoricamente, di Piero Calamandrei ${ }^{[25]}$.

Il potere costituente, lo abbiamo visto, è la prima e l'ultima risorsa a cui il popolo può sempre ricorrere, ma non il popolo indistinto, il popolo agito, quel popolo considerato una massa manovrabile che non delibera, che non è messa in condizione di agire ma è etero-diretta, condizionata e ingannata, nella ricerca costante da parte del leader di una identificazione ideologica (e demagogica $)^{[26]}$.

Il popolo non deve rappresentare un oggetto passivo, di manovra, secondo il linguaggio gramsciano, ma invero il soggetto storico del cambiamento, incarnato (inverato, verrebbe da dire) nelle «esperienza politiche collettive» del Novecento $\stackrel{[27]}{ }$, legittimate, esse sì, a dar vita a un'assemblea o se si preferisce a un processo o a un'esperienza costituente. Quelli che una volta erano i lavoratori rappresentati nei partiti politici e nei sindacati oggi si stenta a identificare. Come è stato ipotizzato, i partiti politici, che avevano svolto il ruolo di raccordo tra società civile e Stato, 
con l'esaurirsi o perlomeno l'attenuarsi della centralità di quest'ultimo, hanno perso la loro rilevanza e, forse, la loro ragion d'essere, proprio nel crepuscolo della statualità ${ }^{[28]}$.

Il progetto incompiuto della modernità costituzionale non pare trovare nuova linfa. Forse proprio la rivendicazione dei "fondamentali" della Costituzione del 1948 - il diritto al lavoro, l'educazione pubblica, laica e gratuita e la dignità dell'uomo - possono orientare il futuro.

Note:

[1] Tra la vastissima letteratura, si seda da ultimo F. Proietti, L'invenzione della democrazia. Pensiero politico e istituzioni nella Seconda Repubblica francese (1848-1852), Aracne, Roma 2020.

[2] Per una sintesi cfr. M. Cotta, Rappresentanza politica, in Dizionario di Politica, diretto da N. Bobbio, N. Matteucci e G. Pasquino, Utet, Torino 1983, pp. 954-959.

[3] $\mathrm{P}$. Bourdieu, La délégation et le fétichisme politique, in «Actes de la recherche en sciences sociales», 52-53 (1984), pp. 49-55.

${ }^{[4]}$ E. Fraenkel, Il doppio Stato. Contributo alla teoria della dittatura (1974), Introduzione di N. Bobbio, Einaudi, Torino 1983.

${ }^{[5]}$ Si veda ampiamento A. Buratti, Dal diritto di resistenza al metodo democratico. Per una genealogia del principio di opposizione nello Stato costituzionale, Giuffrè, Milano 2006.

[6] D. Di Cesare, Il tempo della rivolta, Bollati Boringhieri, Torino 2020, p 30.

${ }^{[7]}$ G. Filippetta, L'estate che imparammo a sparare. Storia partigiana della Costituzione, Feltrinelli, Milano 2018.

${ }^{[8]}$ M. Dogliani, Convenzioni costituenti e forma di governo nella Resistenza e nella transizione costituzionale, in Resistenza e diritto pubblico, a cura di F. Cortese, Firenze University Press, Firenze 2016, pp. 133-161.

[9] S. Curreri, Democrazia e rappresentanza politica: dal divieto di mandato al mandato di partito, Firenze University Press, Firenze 2004; G. Ferrara, Democrazia e rappresentanza politica, in «Costituzionalismo.it», 2005, n. 1.

${ }^{[10]}$ Così L. Del Savio, M. Mameli, Controsovranità. La democrazia oltre la democrazia rappresentativa, Feltrinelli, Milano 2015, pp. 14 ss.

[11] Sul punto si veda ora A.M. Banti, La democrazia dei followers. Neoliberismo e cultura di massa, Laterza, Roma-Bari 2020.

[12] Nell'amplissima bibliografia si veda, da ultimo, l'ottima sintesi offerta da M. Dogliani, C. Giorgi, Art. 3, Carocci, Roma 2017; in un quadro teorico più ampio, L. Ferrajoli, Manifesto per l'uguaglianza, Laterza, Roma-Bari 2018. 
[13] Dogliani, Giorgi, 2017., p. 9.

[14] Secondo quanto scritto da P. Costa, M. Salvati, La serie "Costituzione italiana, Carocci, Roma 2017, pp. XIII ss.

${ }^{[15]}$ C. Cost., sent. n. 1146/1988. Affermazioni ribadite anche nella recente sent. n. 238/2014; sul punto A. Buratti, Art. 139, in La Costituzione italiana. Commento articolo per articolo, 2 voll., a cura di F. Clementi, L. Cuocolo, F. Rosa, G.E. Vigevani, Il Mulino, Bologna 2018.

[16], Contro il revisionismo costituzionale. Tornare ai fondamentali, Laterza, Roma-Bari 2016.

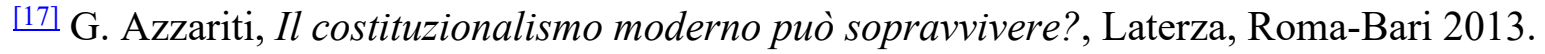

$118]$ P. Scoppola, La Costituzione contesa, Einaudi, Torino 1998, p. 92.

[19] J. Elster, Ulisse liberato. Razionalità e vincoli, Il Mulino, Bologna 2004.

[20] Si veda L. Nivarra, Dalla “crisi" all"”eclissi”: ovvero, da un paradigma all'altro, in «Europa e diritto privato», 3/2017, pp. 801-846.

[21] Sul punto si veda ampiamente A. Burgio, Senza democrazia. Un'analisi della crisi, DeriveApprodi, Roma 2009, pp. 74 ss.

${ }^{[22]}$ Così U. Mattei, Contro riforme, Einaudi, Torino 2013.

${ }^{[23]}$ Cfr. per esempio Invertire la rotta. Idee per una riforma della proprietà pubblica, a cura di U. Mattei, E. Reviglio, S. Rodotà, Il Mulino, Bologna 2007; «Rivista Critica del Diritto Privato», XXX (2012), n. 1, in particolare C. Salvi, Codice civile e Costituzione, pp. 31-40.

[24] Seguendo l'interpretazione fornita da M. D'Eramo, Dominio. La guerra invisibile dei potenti contro $i$ sudditi, Feltrinelli, Milano 2020.

$25]$ Si veda G. Pasquino, La rivoluzione promessa. Lettura della Costituzione italiana, Bruno Mondadori, Milano 2011.

[26] Cfr. G. Zagrebelsky, Il «crucifige!» e la democrazia, Einaudi, Torino 2007; V. Pazé, In nome del popolo. Il problema democratico, Laterza, Roma-Bari 2011, pp. 63-64.

[27] V. Onida, La Costituzione, Il Mulino, Bologna 2007, p. 134.

[28] Così G. De Luna, La Repubblica inquieta. L'Italia della Costituzione. 1946-1948, Feltrinelli, Milano 2017, pp. 148-149. 